\title{
Evaluating the prevalence and severity of NAFLD in primary care: the EPSONIP study protocol
}

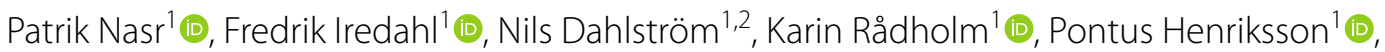 \\ Gunnar Cedersund ${ }^{2,3}$, Olof Dahlqvist Leinhard ${ }^{1,2,4}$, Tino Ebbers ${ }^{1} \mathbb{C}^{0}$, Joakim Alfredsson ${ }^{1}$, Carl-Johan Carlhäll, ${ }^{10}$, \\ Peter Lundberg ${ }^{1,2}$ (D) Stergios Kechagias ${ }^{1}$ (D) and Mattias Ekstedt ${ }^{1,2^{*}}$ (D)
}

\begin{abstract}
Background: Non-alcoholic fatty liver disease (NAFLD) affects 20-30\% of the general adult population. NAFLD patients with type 2 diabetes mellitus (T2DM) are at an increased risk of advanced fibrosis, which puts them at risk of cardiovascular complications, hepatocellular carcinoma, or liver failure. Liver biopsy is the gold standard for assessing hepatic fibrosis. However, its utility is inherently limited. Consequently, the prevalence and characteristics of T2DM patients with advanced fibrosis are unknown. Therefore, the purpose of the current study is to evaluate the prevalence and severity of NAFLD in patients with T2DM by recruiting participants from primary care, using the latest imaging modalities, to collect a cohort of well phenotyped patients.
\end{abstract}

Methods: We will prospectively recruit 400 patients with T2DM using biomarkers to assess their status. Specifically, we will evaluate liver fat content using magnetic resonance imaging (MRI); hepatic fibrosis using MR elastography and vibration-controlled transient elastography; muscle composition and body fat distribution using water-fat separated whole body MRl; and cardiac function, structure, and tissue characteristics, using cardiovascular MRI.

Discussion: We expect that the study will uncover potential mechanisms of advanced hepatic fibrosis in NAFLD and T2DM and equip the clinician with better diagnostic tools for the care of T2DM patients with NAFLD.

Trial registration: Clinicaltrials.gov, identifier NCT03864510. Registered 6 March 2019, https://clinicaltrials.gov/ct2/ show/NCT03864510.

Keywords: Non-alcoholic fatty liver disease, Type 2 diabetes mellitus, T2DM, Cirrhosis, Biomarkers

\section{Background}

Non-alcoholic fatty liver disease (NAFLD) is the most common chronic liver disease, with a worldwide prevalence of $20-30 \%$ [1]. Histological features range from hepatic steatosis to non-alcoholic steatohepatitis (NASH), the latter being characterized by inflammation,

\footnotetext{
*Correspondence: mattias.ekstedt@liu.se

${ }^{1}$ Department of Health, Medicine and Caring Sciences, Linköping University, Linköping, Sweden

Full list of author information is available at the end of the article
}

with or without fibrosis, with the risk of progressing to cirrhosis [2]. Cirrhosis, in turn, is associated with a $2.5 \%$ annual risk of developing hepatocellular carcinoma (HCC) [3]. In the near future, NAFLD is expected to become the leading cause for liver transplantation [4].

NAFLD increases the risk of liver-related and cardiovascular morbidity and mortality $[5,6]$. Clinical and histological variables that predict overall mortality in NAFLD are age, type 2 diabetes mellitus (T2DM) [7, 8], and liver fibrosis $[7,9,10]$. 
In the last four decades, there has been a steep increase in T2DM global prevalence, both in high/ middle-, and low-income countries [11]. And although NAFLD is strongly associated with the metabolic syndrome and T2DM [12], the association is bidirectional; with a markedly higher prevalence of NAFLD in patients with T2DM (40-70\%) than in individuals without T2DM, and an increased incidence of T2DM in patients with NAFLD [13, 14].

Liver biopsy is the current gold standard for diagnosing severity of NAFLD. However, it has several limitations, including adverse events as well as sampling and observer variability [15-17]. Consequently, noninvasive methods are being evaluated to replace liver biopsy. These include magnetic resonance (MR)-based methods and serological marker testing.

Recently, the European Association for the Study of Diabetes (EASD), Obesity (EASO) and the Liver (EASL) proposed non-invasive screening for NAFLD and advanced fibrosis among patients with T2DM [18]. Nevertheless, to date, there is no clear consensus on how to implement these guidelines. Furthermore, concerns have been raised on whether screening is costeffective, especially since most available non-invasive tests have low positive predictive value and because treatment of liver fibrosis is lacking [19].

Hepatic fat can be assessed directly by proton density fat fraction (PDFF) using magnetic resonance techniques [20, 21]. However, hepatic fibrosis has no molecular signature that can be detected and is therefore assessed indirectly by quantification of liver "stiffness" (or "elasticity") [20]. The most accurate noninvasive methods for assessing stiffness include transient elastography (TE) and MR elastography (MRE).

Serological markers for the evaluation of liver fibrosis are more accessible and easier to use than imaging and therefore preferable for the evaluation of a prevalent disease. However, no panel of serological fibrosis markers has shown clinically acceptable sensitivity required for the diagnosis of advanced fibrosis although they can be used to exclude advanced fibrosis [24].

Most NAFLD-studies in patients with T2DM have been performed at tertiary centers where patients are more likely to have advanced NAFLD. In Sweden, most patients with T2DM is cared for in primary care. Hence, hospital series of NAFLD in T2DM patients is misleading. Therefore, we aim to recruit study participants from primary care, using the latest imaging techniques, to collect a cohort of well phenotyped patients with T2DM to evaluate the prevalence and severity of NAFLD in primary care.

\section{Methods/design}

\section{Overview}

The EPSONIP (Evaluating the Prevalence and Severity Of NAFLD in Primary Care) study is a prospective cohort study with the aim to recruit 400 highly phenotyped patients with T2DM from primary care that will facilitate cross-sectional as well as longitudinal and long-term analyses. The comprehensive study protocol includes clinical information, fitness assessment, physical activity in everyday life, magnetic resonance imaging, lifestyle, and quality of life (QoL) data, as well as a biological sample collection (including genetic analysis). Specifically, we will evaluate liver fat content using magnetic resonance imaging (MRI); hepatic fibrosis, using magnetic resonance elastography (MRE) and vibration-controlled transient elastography (VCTE); fat free muscle volume, muscle fat infiltration, and abdominal fat distribution, using whole body fat-water separated MRI; and cardiac function and structure, using cardiovascular MRI (cardiovascular MRI will be assessed in 200 participants). Each participant will be investigated twice, at 3-year intervals, to identify individuals that develop cardiovascular disease and comorbidities, as well as progressive liver disease. See Fig. 1 for an overview of study procedures.

\section{Objectives}

The overall purpose of the study is to better characterize the T2DM-NAFLD patient population using noninvasive methods to inform the diagnosis and care for this population. We aim to do this by identifying the relationship between T2DM, NAFLD, advanced hepatic fibrosis and myocardial dysfunction, as well as clinical, biochemical, and lifestyle parameters of patients with T2DM and NAFLD.

\section{Specific aims}

1. To determine the prevalence and incidence of NAFLD and advanced fibrosis in participants with T2DM using advanced non-invasive approaches.

2. To test whether serological markers enable a faster and better-informed assessment than liver biopsy of the risk of advanced hepatic fibrosis, myocardial fibrosis, or myocardial dysfunction in participants with T2DM.

3. To identify the clinical, biochemical, putative pathophysiological, and genetic factors associated with NAFLD and advanced fibrosis in participants with T2DM 


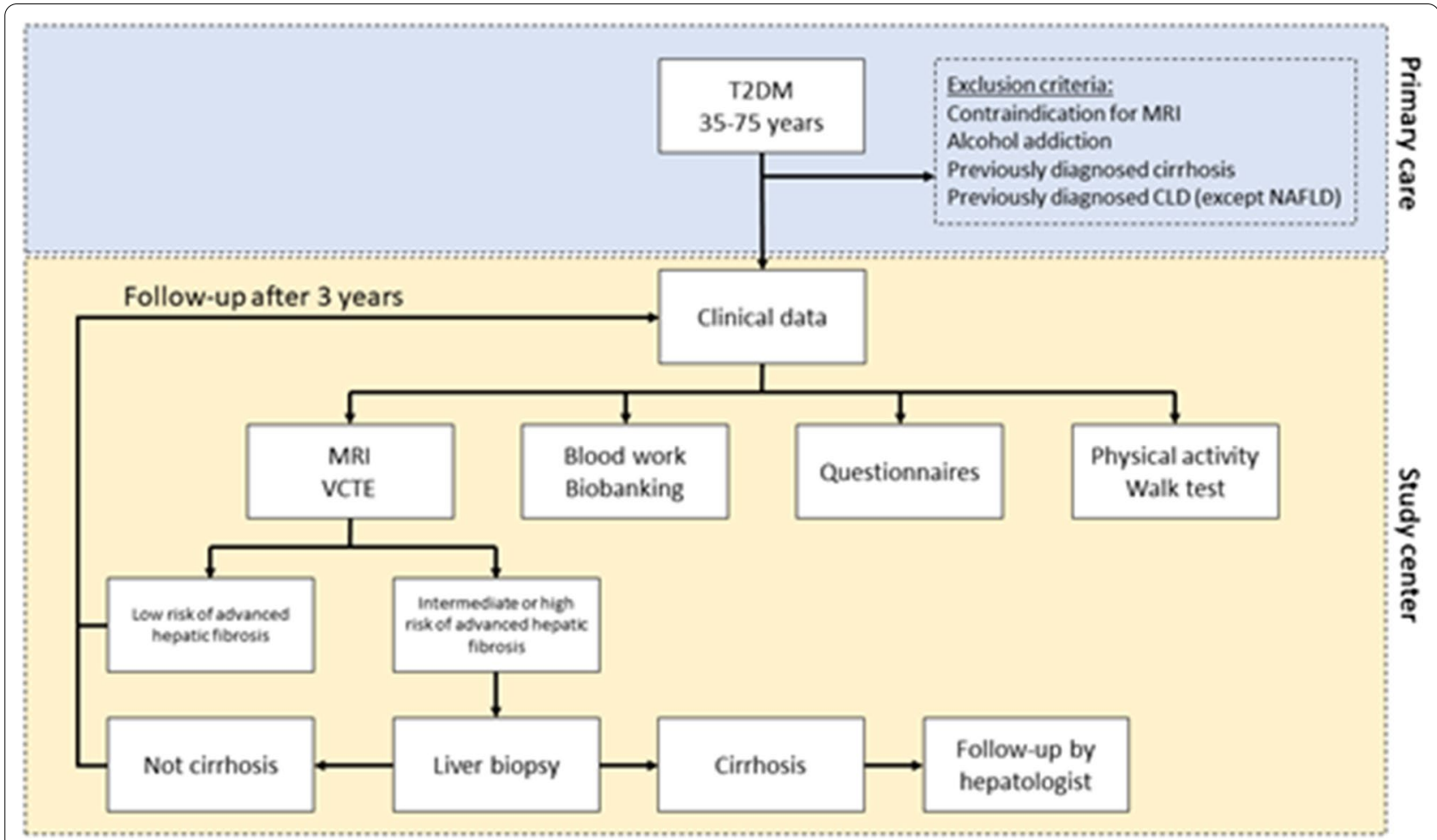

Fig. 1 Study flow chart outlining patient recruitment, inclusion, data collection and follow-up. CLD, chronic liver disease; MRI, magnetic resonance imaging; NAFLD, non-alcoholic fatty liver disease; T2DM, type 2 diabetes mellitus; VCTE, vibration controlled transient elastography

4. To determine the relationship between NAFLD, myocardial function, and clinical, biochemical, and lifestyle factors in patients with and without T2DM.

5. To identify non-invasive biomarkers that predict fibrosis progression and altered myocardial function in NAFLD patients with and without T2DM.

\section{Organization and oversight}

The study is run and coordinated by Dr. Mattias Ekstedt (PI) at the Department of Gastroenterology and Hepatology, University Hospital in Linköping, and Faculty of Medicine and Health Sciences, Linköping University. The study will be monitored by Forum Östergötland.

Patients will be recruited at primary healthcare centers. At present, four healthcare centers in South East Sweden (in Östergötland) are part of the study (Ekholmen and Kärna in Linköping, and Åby, Helsa Vårdcentral Kneippen and Cityhälsan Söder in Norrköping). Patient recruitment at each health care center will be overseen by General Practitioners participating in the study. The PI will supervise activities during regular on-site meetings. Collection of clinical data and blood samples will be performed by experienced research nurses. MRI will be performed at Center for Medical Image Science and
Visualization (CMIV) in Linköping and the Department of Radiology at Vrinnevisjukhuset in Norrköping.

\section{Ethics approval and consent to participate}

All recruitment and attaining written informed consent are conducted according to nationally accepted practice and in full accordance with the World Medical Association of Helsinki 2018. Data is collected and processed in accordance with the applicable General Data Protection Regulation (EU) 2016/679 (GDPR) legislation, and in compliance with the International Conference of Harmonization-Good Clinical Practice (ICH-GCP) requirements [22].

The EPSONIP study was approved by the Regional Ethical Board of Östergötland 2018/176-31 and 2018/494-32 and is registered as a clinical trial (clinicaltrials.gov identifier NCT 03864510). A complementary amendment, titled EPSONIP_Sleep, has been approved by the Swedish Ethical Review Authority 2019-03854.

\section{Participants}

Patients with T2DM attending annual check-ups at their primary healthcare center will be eligible for inclusion in the study. Patients will be invited to participate by their diabetes nurse or treating physician. 
Table 1 Inclusion and exclusion criteria

\begin{tabular}{|c|c|}
\hline \multicolumn{2}{|l|}{ Criteria } \\
\hline \multirow[t]{2}{*}{ Inclusion } & Diagnosis of T2DM according to current guidelines \\
\hline & Age: $35-75$ years \\
\hline \multirow[t]{4}{*}{ Exclusion } & $\begin{array}{l}\text { Contraindications to perform MRI (pacemaker, ferrous metal } \\
\text { implants/fragments, claustrophobia, extreme obesity, and/ } \\
\text { or pregnancy) }\end{array}$ \\
\hline & Alcohol dependence \\
\hline & Previously diagnosed liver cirrhosis \\
\hline & Previously diagnosed primary liver disease (except NAFLD) \\
\hline
\end{tabular}

Patients of both sexes will be consecutively included. The inclusion and exclusion criteria are presented in Table 1

Following receipt of information about the study and the opportunity to ask questions, participants will be asked to provide written informed consent, witnessed, and dated, by a member of the research team. Written informed consent will always be obtained prior to study-specific procedures.

\section{Estimation of sample size and power calculation}

The cohort size was primarily defined so that a significant number of patients with significant or progressive fibrosis was identified. In previous studies of diabetic patients, $45-65 \%$ had fatty liver, and $7 \%$ had significant fibrosis [23]. Extrapolated to our study, that would give 260 patients with fatty liver and 28 patients with advanced fibrosis at baseline. At follow-up, one third of the patients with fatty liver is expected to progress in fibrosis stage. Therefore, we expect that approximately 86 patients will have a progressive disease state.

For the majority of our aims, no power calculation can be performed. But to ascertain that we had significant power to investigate non-invasive markers relevant for this patient cohort we made power calculations for one baseline and one follow-up parameter. Given the focus of the study on ectopic fat accumulation we wanted to ascertain that we had sufficient power to detect a difference in epicardial fat accumulation between diabetic patients with and without fatty liver at baseline. In a previous study performed by our group, patients with diabetes had an epicardial fat volume of $62.1 \pm 21.0 \mathrm{~mL} / \mathrm{m}^{2}$. We expect at least a $10 \%$ difference between groups. The power calculation indicated, with $80 \%$ power, that 90 patients would be sufficient to detect a difference at an $\alpha$ value of 0.05 . For the follow-up assessment we decided to use end diastolic volume (EDV) as a surrogate for cardiac remodeling. In our patients with diabetes the EDV was $69.6 \pm 15.2 \mathrm{~mL} /$
Table 2 Layout of the anthropometric and clinical data collected at inclusion and 3 year follow-up

\begin{tabular}{l}
\hline Categories of data \\
\hline Basic data \\
Date of birth \\
Gender \\
Anthropometrics \\
Height (cm) \\
Weight (kg) \\
Waist circumference (cm) \\
Hip circumference (cm) \\
Blood pressure (mmHg) \\
Medical history \\
Date of T2DM diagnosis \\
Current or recent medication (including over-the-counter, tradi- \\
tional/herbal remedies, and nutritional supplements) \\
Relevant comorbidities and date of diagnosis, including \\
Hypertension, dyslipidemia \\
Ischemic heart disease, including PCl and CABG \\
Congestive heart failure \\
Stroke \\
Malignancies \\
Lifestyle \\
Smoking—yes/no/ex, and frequency of smoking (pack-years) \\
Coffee consumption—cups/days \\
Alcohol consumption \\
Physical activity and fitness \\
Patient reported quality of life \\
Sleep quality assessment \\
Family history \\
Family medical history \\
(c)
\end{tabular}

$\mathrm{m}^{2}$. We expect at least a $10 \%$ difference between groups. The power calculation indicated, with $80 \%$ power, that 146 patients would be sufficient to detect a difference at an $\alpha$ value of 0.05 .

\section{Study procedures}

Following the provision of informed consent, patients will be assigned a unique study-participate identification code incorporating the recruitment site identifier. All data will be link-anonymized throughout the study, recorded through a secure web-based application for electronic data (REDCap ${ }^{\mathrm{TM}}$ ).

Patients that have consented to participate in the study will participate in a subsequent study visit at one of two sites (Linköping or Norrköping). A member of the research team will complete a clinical report form on clinical data (Table 2), with special focus on T2DM history and treatment. Questionnaires regarding the lifestyle and self-reported quality of life will be obtained, 
fitness and physical activity will be assessed, and baseline clinical biochemistry will be obtained.

\section{Questionnaires}

Four validated questionnaires will be used to assess lifestyle factors relevant to NAFLD:

1. Alcohol questionnaires

- AUDIT: The Alcohol Use Disorders Identification Test (AUDIT), a screening tool developed by the World Health Organization to assess alcohol consumption, drinking behaviors, and alcohol-related problems [24].

- LDH: Lifetime Drinking History, designed to provide quantitative indices of an individual's alcohol consumption patterns from the onset of regular drinking [25].

2. Lifestyle questionnaire

- Dietary and physical activity variables are assessed using the validated questionnaires developed by the National Board of Health and Welfare in Sweden $[26,27]$.

- IFIS: The International Fitness Scale, to assess selfreported fitness [28].

- ESS: Epworth Sleepiness Scale [29].

- PSQI: Pittsburgh Sleep Quality Index [30].

- STOP-Bang: Obstructive sleep apnea questionnaire [31].

3. Patient-reported quality of life.

- EQ-5D-5L: This questionnaire was developed by the EuroQol Group in 2009 as a measure of healthrelated quality of life. The descriptive system comprises five dimensions: mobility, self-care, usual activities, pain/discomfort, and anxiety/depression [32].

\section{Fitness and physical activity}

Lifestyle and NAFLD are closely related. Accordingly, we will perform an objective cardiorespiratory fitness measurement (6-min walk test) and a 7 day registration of objective physical activity using an accelerometer (Actigraph ${ }^{\circledR}$ GT3X, Pensacola, FL, USA).

- The 6-min walk test is a submaximal functional exercise test that measures the distance walked over a period of $6 \mathrm{~min}$ [33]. The 6-min walk distance provides a measure for integrated global response of multiple cardiopulmonary and musculoskeletal sys- tems involved in exercise, i.e., cardiorespiratory fitness.

- Physical activity over $7 \mathrm{~d}$ will be recorded using Actigraph ${ }^{\circledR}$ GT3X, a small non-invasive accelerometer worn on the wrist, that captures and records continuous, high-resolution physical activity and sleep/ wake information.

- One overnight registration of signs related to obstructive sleep apnea recorded by home respiratory polygraphy.

\section{Blood sample acquisition and biorepository}

Each patient will be asked to provide blood and urine samples that will be physically stored at the Linköping Biobank Facility (Fig. 2). The biobank facility is a collaboration between the Faculty of Medicine and Health Sciences at Linköping University and Region Östergötland, with a state-of-the-art facility for quality-controlled storage in secure freezers. All samples are collected after an overnight fast. The following amounts of blood and urine will be collected from each patient:

- $3 \times 5 \mathrm{~mL}$, into EDTA Vacutainer tubes for plasma collection, for subsequent analyses of fibrosis biomarkers and metabolomics/proteomics/lipidomics

- $4 \times 5 \mathrm{~mL}$, into red top Vacutainer tubes for serum collection, for subsequent analyses of fibrosis biomarkers or metabolomics/proteomics/lipidomics

- $1 \times 5$ mL, into PAX tube for RNA preservation, for blood RNA extraction for transcriptomic analysis

- $1 \times 10 \mathrm{~mL}$, into PAX tube, for extraction of circulating cell-free DNA

- $1 \times 100 \mathrm{~mL}$ urine into a sterile collecting vial, after centrifugation urine pellet is collected into a $2 \mathrm{ml}$ cryovial and urine is stored in $12 \times 4 \mathrm{ml}$ cryovials

\section{Clinical hematology/biochemistry/immunology/virology}

Several blood, serum and plasma markers will be analyzed (Table 3) to exclude other causes of chronic liver disease, obtain a metabolic profile and to calculate previously identified biomarker algorithms associated with advanced fibrosis (Additional file 1: Table S1).

\section{Multimodal MR examination}

We have devised a multimodal MR-protocol for this project that includes a range of specific MR-techniques, including determination of iron concentrations in the liver, PDFF determination using MRS to measure hepatic triglyceride concentration (Fig. 3a, b), and 3D-MRE to determine the hepatic fibrosis stage (Fig. 3c). The cardiac investigations will include cine morphological MRI, 


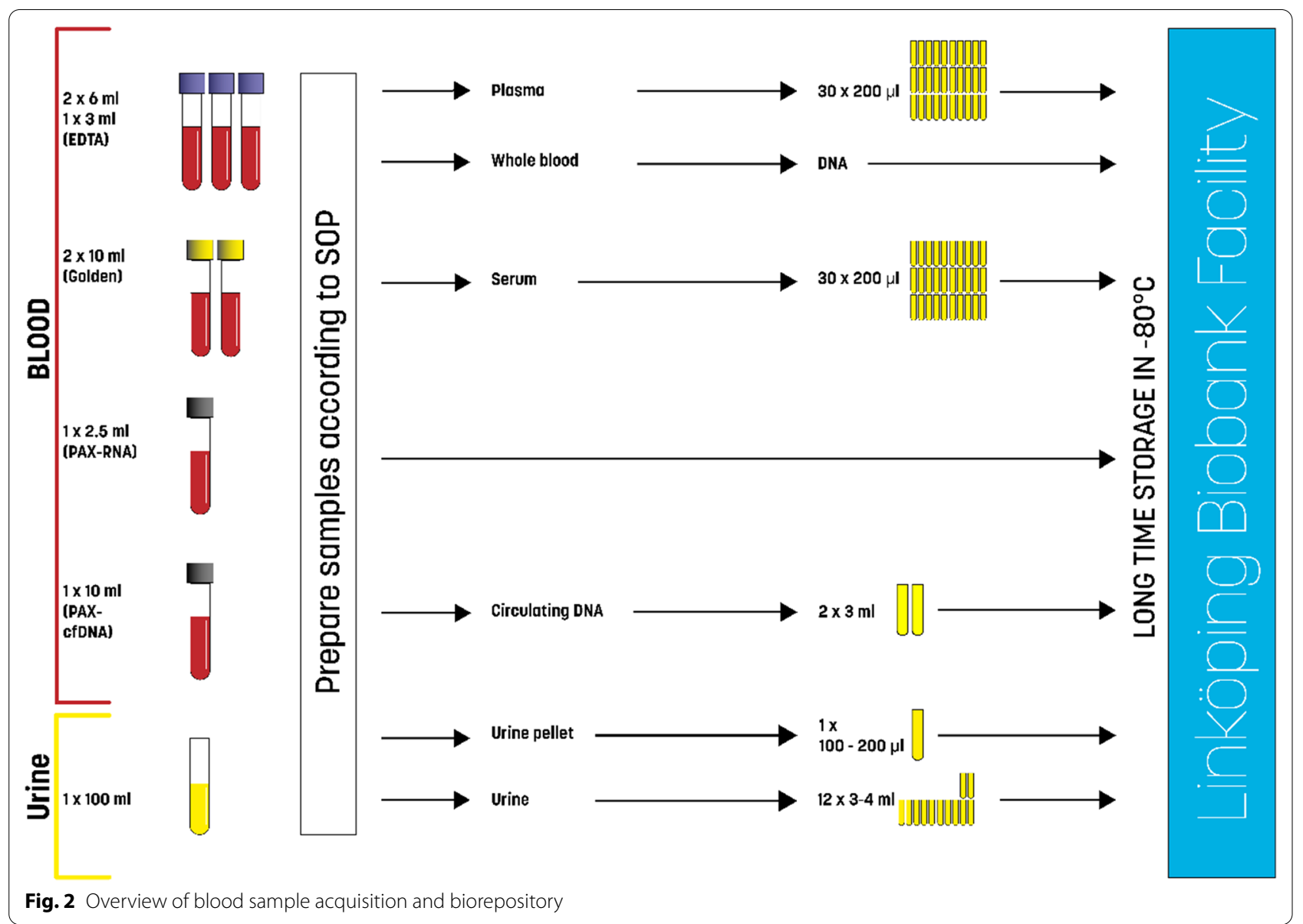

a reference method for volumetric assessment, including left ventricular size, stroke volume and ejection fraction; native 3D-QALAS for 3D mapping of T1 and T2 relaxation time in the whole myocardium in one breath-hold, to estimate diffuse myocardial fibrosis; cardiac DIXON imaging, to determine epi- and paracardial adipose tissue; and 4D flow MRI, for the assessment of blood flow and model-based assessment of cardiac function (Fig. 3e). Furthermore, the protocol will include whole body waterfat separated imaging for quantification of visceral and subcutaneous adipose tissue volume, fat free thigh muscle volume, and muscle fat infiltration (Fig. 3d). The protocol will be used for data acquisition on $1.5 \mathrm{~T}$ MRscanners (Philips Healthcare, Best, The Netherlands) in both Linköping (at CMIV) and Norrköping. The protocol is efficiently condensed, and the data will be acquired within $50 \mathrm{~min}$.

\section{Vibration-controlled transient elastography (VCTE)}

Transient elastography (TE) relies on a transient mechanical vibration used to induce a shear wave in a tissue. The propagation of the shear wave is then tracked using ultrasound to assess the shear wave speed. A specific implementation of 1D-TE, vibration controlled TE (VCTE), has been developed to assess the average liver stiffness that correlates with liver fibrosis assessed by liver biopsy [33]. In this study, it will be implemented using FibroScan ${ }^{\circledR}$, which is available at both project sites (Linköping and Norrköping), including $\mathrm{M}$ - and XL-probe as well as Controlled attenuation parameter (CAP). CAP measures liver ultrasonic attenuation on the signals acquired by the FibroScan ${ }^{\circledR}$. Principles of CAP measurements has been described elsewhere [34].

\section{Liver biopsy}

Patients with increased values of Fibroscan ${ }^{\circledR}$ or MRE will be offered a liver biopsy to confirm the fibrosis stage. Liver biopsies will be performed according to the clinical routine at the University Hospital in Linköping. All biopsies will be performed with ultrasound guidance, using a 1.6-mm Biopince ${ }^{\circledR}$ needle. A pathologist with NAFLD experience will assess the biopsies. The following histopathological variables will be recorded: steatosis, lobular inflammation, hepatocellular 
Table 3 Detailed blood/serum/plasma work-up

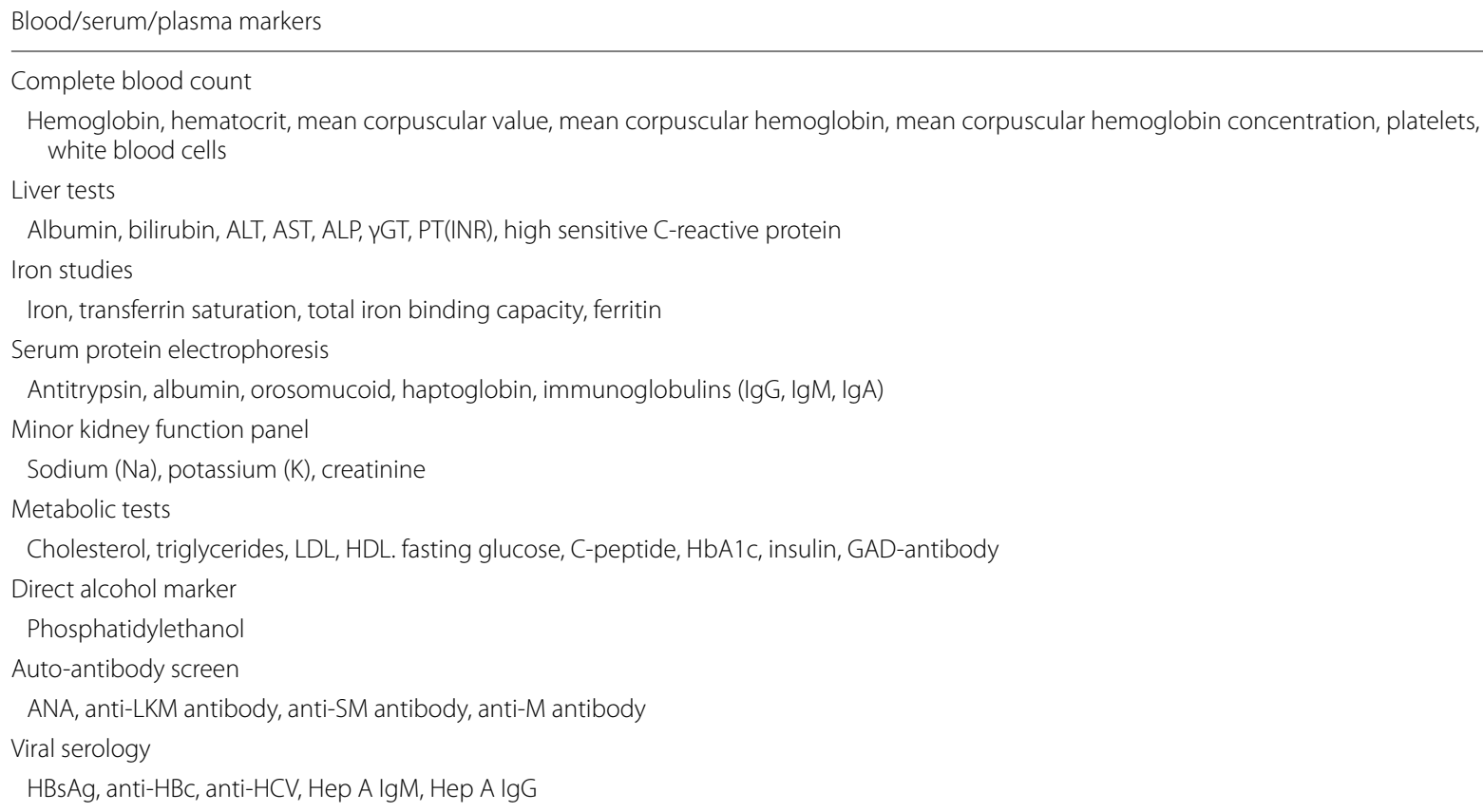

ALP, alkaline phosphatase; ALT, alanine aminotransferase; ANA, antinuclear antibody; AST, aspartate aminotransferase; GAD, glutamic acid decarboxylase; $\gamma$ GT, gamma-glutamyl transferase; $\mathrm{HbA1C}$, hemoglobin $\mathrm{A} 1 \mathrm{c} ; \mathrm{HBC}$, hepatitis B core; $\mathrm{HBs} A$ g, hepatitis B surface antigen; $\mathrm{HCV}$, hepatitis $\mathrm{C}$ virus; $\mathrm{HDL}$, high-density lipoprotein; INR, international normalized ratio; LKM, liver-kidney microsomal; LDL, low-density lipoprotein; M, mitochondrial; PT, prothrombin time; SM, smooth muscle

ballooning, portal inflammation, Mallory-Denk bodies, and portal/pericellular fibrosis. The pathologist will also perform a global assessment for the presence of NASH [35].

\section{3-Year follow-up}

Follow-up will be performed 3 years after the initial examination to assess the progression or regression of incidence of NAFLD and advanced hepatic fibrosis, as well as development of cardiovascular and liver-related events. A complete list of clinically significant events is presented in Table 4. The follow-up protocol is planned to be identical to the baseline protocol, although minor changes may be done after a renewed ethical review due to development within the research field.

\section{Time plan and implementation}

The project involves patient recruitment, data acquisition, and data analysis. Patient recruitment started in March 2019. The goal is to complete patient inclusion in 2022.

In terms of data acquisition, the MR-scanners have been operational since March 2020.

Repeated 3-year MR imaging is expected to be concluded in 2026.

\section{Discussion}

NAFLD is the most prevalent liver disease worldwide and is strongly associated with increased mortality from cardiovascular disease (CVD). NAFLD is rapidly becoming the leading cause of advanced liver disease in Western countries, and is the main reason for liver transplantation [36-38]. Albeit being highly prevalent, only a minority of patients with NAFLD (4-10\%, depending on the followup time) will progress to cirrhosis and end stage liver disease [39]. However, because of its high heterogeneity, it is challenging to identify NAFLD patient at risk of progression [40]. Although fibrosis stage is a robust predictor of liver related morbidity and all-cause mortality in patient with NAFLD [6], the use of liver biopsy in a routine clinical setting, for a highly prevalent disease, is not realistic. Therefore, noninvasive modalities for the diagnosis of advanced fibrosis have been proposed. Magnetic resonance elastography and vibration controlled transient elastography are two highly validated systems with high negative predictive values but low positive predictive values for the detection of advanced hepatic fibrosis. A low value obtained with MRE or VCTE excludes advanced fibrosis with high precision.

The burden of T2DM is at an all-time high, expected to increase in parallel with the obesity pandemic [11, 41]. The hallmark, i.e. insulin resistance, does not only result in development of T2DM, but also NAFLD; therefore, the 

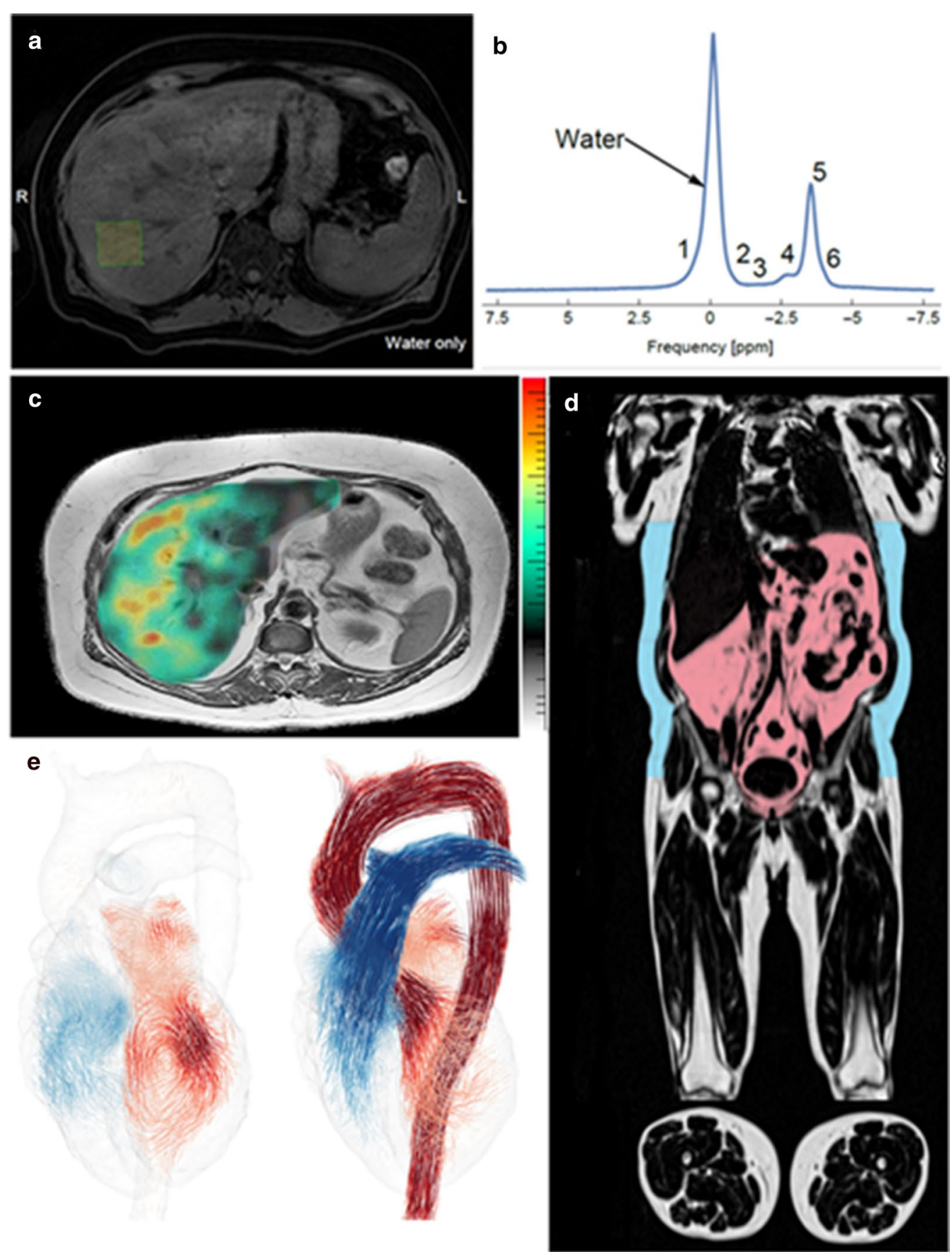

Fig. 3 Image A shows the representative water MR image with the placement of a proton magnetic resonance spectroscopy ( $\left.{ }^{1} \mathrm{H}-\mathrm{MRS}\right)$ voxel in the right hepatic lobe. Image B shows in vivo ${ }^{1} \mathrm{H}$-MRS spectrum for water and fat. Image C shows MRE for a cirrhotic NAFLD patient. Image E shows a whole-body water-fat separated imaging for quantification of visceral and subcutaneous adipose tissue volume. And image D shows a 4D flow image of a healthy heart. 4D flow, four-dimensional flow; ${ }^{1} \mathrm{H}-\mathrm{MRS}$, proton magnetic resonance spectroscopy; MR, magnetic resonance; MRE, MR elastography

three conditions are seen as intertwined [42, 43]. Furthermore, T2DM has shown to predict progression to severe liver disease and development of HCC in individuals with NAFLD [44]. This spurred the EASL, EASO and EASD to recommend screening for the presence of hepatic fat and advanced fibrosis in individuals with T2DM [45]. The characteristics of T2DM patients with advanced hepatic fibrosis are unknown, as are the potential mechanisms of 
Table 4 Clinically significant events registered at follow-up after 3 years of follow-up

\begin{tabular}{l}
\hline Event category \\
\hline Death \\
Cause of death \\
Major Cardiovascular Event \\
Non-fatal stroke \\
Non-fatal myocardial infarction \\
Coronary revascularization \\
Hospitalisation for heart failure \\
Atrial fibrillation \\
Hepatic \\
Diagnosis of cirrhosis \\
Diagnosis of any cirrhosis complication \\
Varices or variceal haemorrhages \\
Ascites \\
Encephalopathy \\
Hepatocellular carcinoma \\
Liver transplantation \\
\hline
\end{tabular}

advanced hepatic fibrosis in NAFLD and T2DM, and factors that potentiate the development of health complications in T2DM patients with NAFLD.

Moreover, NAFLD is an important cardiovascular risk factor [46, 47]. However, the relationship between NAFLD and myocardial fibrosis and dysfunction in participants with T2DM is poorly understood. One important factor is the dysregulation of altered hormonal gene regulation associated with NAFLD, with increased proatherogenic inflammatory markers, procoagulant factors, and disrupted metabolic equilibrium [46]. Hence, the accelerated atherogenesis in individuals with NAFLD probably has its origin in the visceral and hepatic lipid accumulation, with the liver being both the target of the resulting systemic abnormalities and a source of pro-atherogenic molecules that amplify the arterial damage and alter cardiac structure $[48,49]$. Furthermore, cardiovascular disease and mortality is prevalent among patients with NAFLD, where the risk of incident CVD and the risk of developing CVD is independently increased $[50,51]$. Similarly, the need for coronary angiography, myocardial fibrosis and percutaneous coronary intervention is more common in patients with NAFLD [48, 52, 53]. However, albeit individuals with NAFLD are more prone to a dismal cardiometabolic risk profile, hepatic steatosis is not independently associated with CVD [54, 55]. Therefore, the relationship between NAFLD and CVD is poorly understood, with few predictive markers identified.

Most studies evaluating the outcomes of NAFLD patients have been performed at university hospitals where the referred patients are more likely to have advanced NAFLD and are not necessarily representative of the "general" NAFLD patient population. Therefore, in this prospective study, the 'Evaluating Prevalence and Severity Of NAFLD In Primary care' (EPSONIP) trial (ClinicalTrials.gov Protocol Record 2018:176-31), we will recruit participants from primary care, where the vast majority of NAFLD and T2DM patients are managed in Sweden. We propose to evaluate the utility of advanced non-invasive imaging approaches and serum biomarkers in assessing advanced hepatic fibrosis, myocardial fibrosis, or myocardial dysfunction in patients with T2DM. We anticipate that development of reliable non-invasive methods to diagnose hepatic and myocardial fibrosis proposed herein will enable timely identification of patients with NAFLD and T2DM, at risk of developing future complications. Identification of these patients will allow early prevention, offering evolving pharmacological therapies, and providing monitoring and treatment of life-threatening complications. Unnecessary follow-up will thus be avoided in patients at low risk of developing future complications. In the long term, this will improve the care and quality of life of the affected individuals, and spare costs to healthcare providers as well.

\begin{abstract}
Abbreviations
AUDIT: Alcohol use disorder identification test; CAP: Controlled attenuation parameter; CMIV: Center for Medical Image Science and Visualization; CVD: Cardiovascular disease; EASD: European Association for the Study of Diabetes; EASL: EAS the liver; EASO: EAS obesity; EPSONIP: Evaluation the Prevalence and Severity of NAFLD in Primary care; GDPR: General data protection regulation; HCC: Hepatocellular carcinoma; ICH-GCP: International Council for Harmonisation of Technical Requirements for Pharmaceuticals for Human Use-Good Clinical Practice; IFIS: International fitness scale; LDH: Lifetime drinking history; MR: Magnetic resonance; MRE: MR elastography; MRI: MR imaging; MRS: Magnetic resonance spectroscopy; NAFLD: Non-alcoholic fatty liver disease; NASH: Non-alcoholic steatohepatitis; PDFF: Proton density fat fraction; T2DM: Type 2 diabetes mellitus; TE: Transient elastography; QoL: Quality of life; VCTE: Vibration controlled TE.
\end{abstract}

\section{Supplementary Information}

The online version contains supplementary material available at https://doi. org/10.1186/s12876-021-01763-z.

Additional file 1. Supplementary tables.

\section{Acknowledgements}

Forum Östergötland for assisting in study planning, legal advise and monitoring of data quality. Coordinating study nurses Carola Fagerström (Linköping) and Åsa Stahre Wiberg (Norrköping).

\section{Authors' contributions}

Study concept and design: Conceptualization, funding acquisition and supervision: PN, ND, ODL, TE, JA, CJC, PL, SK, ME; project administration and curation: ME, FI, PL SK; Writing — original draft: PN, SK, ME; Patient recruitment PN, FI, KR, SK, ME. First draft PN, SK, ME. Constructive reading of the manuscript and approval of the final version PN, FI, ND, KR, PH, GC, ODL, TE, JA, CJC, PL, SK, ME. All authors read and approved the final manuscript. 


\section{Funding}

Open access funding provided by Linköping University. ALF Grants, Region Östergötland (ME, FI, PL, PN), non-restricted grants by GILEAD (ME) and Diapharma (SK), Lion Research Grant, Faculty of Medicine, Linköping University (PN), The Swedish Research Council (VR 2020-04826) (PL). None of the funders have any role in the design or conduct of the study.

\section{Availability of data and materials}

The dataset during and/or analysed during the current study available from the corresponding author on reasonable request.

\section{Declarations}

\section{Ethics approval and consent to participate}

All recruitment and attained written informed consent are conducted according to World Medical Association of Helsinki 2018. Data is collected in accordance with the applicable General Data Protection Regulation (EU) 2016/679 (GDPR) legislation, and in compliance with the International Conference of Harmonization—Good Clinical Practice (ICH-GCP) requirements [22]. The EPSONIP study was approved by the Regional Ethical Board 2018/176-31 and 2018/494-32 and is registered as a clinical trial (clinicaltrials.gov identifier NCT 03864510). A complementary amendment, titled EPSONIP_Sleep, has been approved by the Swedish Ethical Review Authority 2019-03854.

\section{Consent for publication}

Not applicable.

\section{Competing interests}

The authors declare that they have no competing interests.

\begin{abstract}
Author details
${ }^{1}$ Department of Health, Medicine and Caring Sciences, Linköping University, Linköping, Sweden. ${ }^{2}$ Center for Medical Image Science and Visualization (CMIV), Linköping University, Linköping, Sweden. ${ }^{3}$ Department of Biomedical Engineering, Linköping University, Linköping, Sweden. ${ }^{4}$ AMRA Medical $A B$, Linköping, Sweden. ${ }^{5}$ Department of Clinical Physiology in Linköping, Department of Health, Medicine and Caring Sciences, Linköping University, Linköping, Sweden.
\end{abstract}

Received: 19 February 2021 Accepted: 12 April 2021 Published online: 20 April 2021

\section{References}

1. Younossi ZM, Koenig AB, Abdelatif D, Fazel Y, Henry L, Wymer M. Global epidemiology of nonalcoholic fatty liver disease-meta-analytic assessment of prevalence, incidence, and outcomes. Hepatology. 2016;64:73-84.

2. Diehl AM, Day C. Cause, pathogenesis, and treatment of nonalcoholic steatohepatitis. N Engl J Med. 2017;377:2063-72.

3. Ascha MS, Hanouneh IA, Lopez R, Tamimi TA, Feldstein AF, Zein NN. The incidence and risk factors of hepatocellular carcinoma in patients with nonalcoholic steatohepatitis. Hepatology. 2010;51:1972-8.

4. Pais R, Barritt AS, Calmus Y, Scatton O, Runge T, Lebray P, Poynard T, et al. NAFLD and liver transplantation: Current burden and expected challenges. J Hepatol. 2016;65:1245-57.

5. Hagström H, Nasr P, Ekstedt M, Hammar U, Stål P, Hultcrantz R, Kechagias S. Fibrosis stage but not NASH predicts mortality and time to development of severe liver disease in biopsy-proven NAFLD. J. Hepatol. 2017; Accepted article.

6. Taylor RS, Taylor RJ, Bayliss S, Hagström H, Nasr P, Schattenberg JM, Ishigami $\mathrm{M}$, et al. Association between fibrosis stage and outcomes of patients with nonalcoholic fatty liver disease: a systematic review and meta-analysis. Gastroenterology. 2020;158(1611-1625):e1612.

7. Angulo P, Kleiner DE, Dam-Larsen S, Adams LA, Bjornsson ES, Charatcharoenwitthaya P, Mills PR, et al. Liver fibrosis, but no other histologic features, is associated with long-term outcomes of patients with nonalcoholic fatty liver disease. Gastroenterology. 2015;149(389-397):e310.
8. Stepanova M, Rafiq N, Makhlouf H, Agrawal R, Kaur I, Younoszai Z, McCullough A, et al. Predictors of all-cause mortality and liver-related mortality in patients with non-alcoholic fatty liver disease (NAFLD). Dig Dis Sci. 2013;58:3017-23.

9. Ekstedt M, Hagström H, Nasr P, Fredrikson M, Stål P, Kechagias S, Hultcrantz R. Fibrosis stage is the strongest predictor for disease-specific mortality in NAFLD after up to 33 years of follow-up. Hepatology. 2015;61:1547-54

10. Dulai PS, Singh S, Patel J, Soni M, Prokop LJ, Younossi Z, Sebastiani G, et al. Increased risk of mortality by fibrosis stage in non-alcoholic fatty liver disease: systematic review and meta-analysis. Hepatology 2017.

11. Cho N, Shaw J, Karuranga S, Huang Y, da Rocha FJ, Ohlrogge A, Malanda B. IDF Diabetes Atlas: Global estimates of diabetes prevalence for 2017 and projections for 2045. Diabetes Res Clin Pract. 2018;138:271-81.

12. Yki-Järvinen $\mathrm{H}$. Non-alcoholic fatty liver disease as a cause and a consequence of metabolic syndrome. Lancet Diabetes Endocrinol. 2014;2:901-10.

13. Anstee QM, Targher G, Day CP. Progression of NAFLD to diabetes mellitus, cardiovascular disease or cirrhosis. Nat Rev Gastroenterol Hepatol. 2013;10:330-44.

14. Nasr P, Fredrikson M, Ekstedt M, Kechagias S. The amount of liver fat predicts mortality and development of type 2 diabetes in non-alcoholic fatty liver disease. Liv. Int. 2020.

15. Piccinino F, Sagnelli E, Pasquale G, Giusti G, Battocchia A, Bernardi M, Bertolazzi R, et al. Complications following percutaneous liver biopsy: a multicentre retrospective study on 68276 biopsies. J Hepatol. 1986;2:165-73.

16. Vuppalanchi R, Ünalp A, Van Natta ML, Cummings OW, Sandrasegaran KE, Hameed T, Tonascia J, et al. Effects of liver biopsy sample length and number of readings on sampling variability in nonalcoholic fatty liver disease. Clin Gastroenterol Hepatol. 2009;7:481-6.

17. Ratziu V, Charlotte F, Heurtier A, Gombert S, Giral P, Bruckert E, Grimaldi A et al. Sampling variability of liver biopsy in nonalcoholic fatty liver disease. Gastroenterology. 2005;128:1898-906.

18. European Association for the Study of the L, European Association for the Study of D, European Association for the Study of O. EASL-EASD-EASO Clinical Practice Guidelines for the management of non-alcoholic fatty liver disease. J Hepatol 2016;64:1388-1402.

19. Chalasani N, Younossi Z, Lavine JE, Diehl AM, Brunt EM, Cusi K, Charlton $M$, et al. The diagnosis and management of non-alcoholic fatty liver disease: practice Guideline by the American Association for the Study of Liver Diseases, American College of Gastroenterology, and the American Gastroenterological Association. Hepatology. 2012;55:2005-23.

20. Dulai PS, Sirlin CB, Loomba R. MRI and MRE for non-invasive quantitative assessment of hepatic steatosis and fibrosis in NAFLD and NASH: clinical trials to clinical practice. J Hepatol. 2016;65:1006-16.

21. Nasr P, Forsgren MF, Ignatova S, Dahlström N, Cedersund G, Leinhard OD, Norén $B$, et al. Using a $3 \%$ proton density fat fraction as a cut-off value increases sensitivity of detection of hepatic steatosis, based on results from histopathology analysis. Gastroenterology. 2017;153(53-55):e57

22. (EMA) EMA. ICH E6 (R2) Good clinical practice. In. https://www.ema. europa.eu/en/ich-e6-r2-good-clinical-practice; 2016.

23. Doycheva I, Cui J, Nguyen P, Costa EA, Hooker J, Hofflich H, Bettencourt $R$, et al. Non-invasive screening of diabetics in primary care for NAFLD and advanced fibrosis by MRI and MRE. Aliment Pharmacol Ther. 2016:43:83-95.

24. Bush K, Kivlahan DR, McDonell MB, Fihn SD, Bradley KA. The AUDIT alcohol consumption questions (AUDIT-C): an effective brief screening test for problem drinking. Ambulatory Care Quality Improvement Project (ACQUIP). Alcohol Use Disorders Identification Test. Arch Intern Med 1998:158:1789-1795.

25. Skinner HA, Sheu W-J. Reliability of alcohol use indices. The Lifetime Drinking History and the MAST. J. Stud. Alcohol 1982;43:1157-1170.

26. Socialstyrelsen. Utdrag ur indikatorbilagan för Nationella riktlinjer för sjukdomsförebyggande metoder 2011. https://www.socialstyrelsen.se/ globalassets/sharepoint-dokument/artikelkatalog/ovrigt/2020-2-6596. pdf; 2020.

27. Olsson SJ, Ekblom Ö, Andersson E, Börjesson M, Kallings LV. Categorical answer modes provide superior validity to open answers when asking for level of physical activity: a cross-sectional study. Scand J Public Health. 2016:44:70-6 
28. Ortega FB, Ruiz JR, España-Romero V, Vicente-Rodriguez G, MartínezGómez D, Manios Y, Béghin L, et al. The International Fitness Scale (IFIS): usefulness of self-reported fitness in youth. Int J Epidemiol. 2011:40:701-11.

29. Johns MW. A new method for measuring daytime sleepiness: the Epworth sleepiness scale. Sleep. 1991;14:540-5.

30. Buysse DJ, Reynolds CF III, Monk TH, Berman SR, Kupfer DJ. The Pittsburgh Sleep Quality Index: a new instrument for psychiatric practice and research. Psychiatry Res. 1989;28:193-213.

31. Chung F, Yegneswaran B, Liao P, Sharon, Vairavanathan S, Islam S, Khajehdehi A, et al. STOP questionnaire. Anesthesiology. 2008;108:812-21.

32. Herdman M, Gudex C, Lloyd A, Janssen M, Kind P, Parkin D, Bonsel G, et al. Development and preliminary testing of the new five-level version of EQ-5D (EQ-5D-5L). Qual Life Res. 2011;20:1727-36.

33. ATS Statement. Am J Respir Crit Care Med. 2002;166:111-7.

34. Sasso M, Beaugrand M, De Ledinghen V, Douvin C, Marcellin P, Poupon $\mathrm{R}$, Sandrin $\mathrm{L}$, et al. Controlled Attenuation Parameter (CAP): a novel VCTE ${ }^{T M}$ guided ultrasonic attenuation measurement for the evaluation of hepatic steatosis: preliminary study and validation in a cohort of patients with chronic liver disease from various causes. Ultrasound Med Biol. 2010;36:1825-35.

35. Chalasani N, Younossi Z, Lavine JE, Charlton M, Cusi K, Rinella M, Harrison SA, et al. The diagnosis and management of nonalcoholic fatty liver disease: Practice guidance from the American Association for the Study of Liver Diseases. Hepatology. 2018;67:328-57.

36. Wong RJ, Aguilar M, Cheung R, Perumpail RB, Harrison SA, Younossi ZM, Ahmed A. Nonalcoholic steatohepatitis is the second leading etiology of liver disease among adults awaiting liver transplantation in the United States. Gastroenterology. 2015;148:547-55.

37. Younossi Z, Stepanova M, Ong JP, Jacobson IM, Bugianesi E, Duseja A, Eguchi Y, et al. Nonalcoholic steatohepatitis is the fastest growing cause of hepatocellular carcinoma in liver transplant candidates. Clin Gastroenterol Hepatol. 2019;17(748-755):e743.

38. Goldberg D, Ditah IC, Saeian K, Lalehzari M, Aronsohn A, Gorospe EC, Charlton M. Changes in the prevalence of hepatitis C virus infection, nonalcoholic steatohepatitis, and alcoholic liver disease among patients with cirrhosis or liver failure on the waitlist for liver transplantation. Gastroenterology. 2017;152(1090-1099):e1091.

39. Kechagias S, Nasr P, Blomdahl J, Ekstedt M. Established and emerging factors affecting the progression of nonalcoholic fatty liver disease. Metabolism. 2020;2010:154183.

40. Nasr P, Ignatova S, Kechagias S, Ekstedt M. Natural history of nonalcoholic fatty liver disease: a prospective follow-up study with serial biopsies. Hepatol Commun. 2018;2:199-210.

41. Collaboration NCDRF. Worldwide trends in body-mass index, underweight, overweight, and obesity from 1975 to 2016: a pooled analysis of 2416 population-based measurement studies in 128.9 million children, adolescents, and adults. Lancet 2017;390:2627-42.

42. Li L, Liu DW, Yan HY, Wang ZY, Zhao SH, Wang B. Obesity is an independent risk factor for non-alcoholic fatty liver disease: evidence from a metaanalysis of 21 cohort studies. Obes Rev. 2016;17:510-9.
43. Loomba R, Sanyal AJ. The global NAFLD epidemic. Nat Rev Gastroenterol Hepatol. 2013;10:686-90.

44. Nasr P, Blomdahl J, Kechagias S, Ekstedt M. Modifiers of liver-related manifestation in the course of NAFLD. Curr Pharm Des. 2020;26:1062-78.

45. Thursz M, Gual A, Lackner C, Mathurin P, Moreno C, Spahr L, Sterneck M, et al. EASL clinical practice guidelines: management of alcohol-related liver disease. J Hepatol. 2018;69:154-81.

46. Targher G, Byrne CD, Tilg H. NAFLD and increased risk of cardiovascular disease: clinical associations, pathophysiological mechanisms and pharmacological implications. Gut 2020:gutjnl-2020-2320.

47. Hagström H, Nasr P, Ekstedt M, Hammar U, Stål P, Askling J, Hultcrantz R, et al. Cardiovascular risk factors in non-alcoholic fatty liver disease. Liver International 2018.

48. Cassidy S, Hallsworth K, Thoma C, Macgowan GA, Hollingsworth KG, Day CP, Taylor R, et al. Cardiac structure and function are altered in type 2 diabetes and Non-alcoholic fatty liver disease and associate with glycemic control. 2015;14:23.

49. Houghton D, Zalewski P, Hallsworth K, Cassidy S, Thoma C, Avery L, Slomko J, et al. The degree of hepatic steatosis associates with impaired cardiac and autonomic function. J. Hepatol. 2019.

50. Wu S, Wu F, Ding Y, Hou J, Bi J, Zhang Z. Association of non-alcoholic fatty liver disease with major adverse cardiovascular events: a systematic review and meta-analysis. Sci Rep. 2016;6:33386

51. Bhatia LS, Curzen NP, Calder PC, Byrne CD. Non-alcoholic fatty liver disease: a new and important cardiovascular risk factor? Eur Heart J. 2012;33:1190-200.

52. Wong VW, Wong GL, Yeung JC, Fung CY, Chan JK, Chang ZH, Kwan CT, et al. Long-term clinical outcomes after fatty liver screening in patients undergoing coronary angiogram: a prospective cohort study. Hepatology. 2016;63:754-63.

53. Fracanzani AL, Tiraboschi S, Pisano G, Consonni D, Baragetti A, Bertelli C, Norata D, et al. Progression of carotid vascular damage and cardiovascular events in non-alcoholic fatty liver disease patients compared to the general population during 10 years of follow-up. Atherosclerosis. 2016;246:208-13.

54. Linge J, Borga M, West J, Tuthill T, Miller MR, Dumitriu A, Thomas EL, et al. Body composition profiling in the UK Biobank Imaging Study. Obesity. 2018:26:1785-95.

55. Shah RV, Anderson A, Ding J, Budoff M, Rider O, Petersen SE, Jensen MK, et al. Pericardial, but not hepatic, fat by CT is associated with CV outcomes and structure: the multi-ethnic study of atherosclerosis. JACC Cardiovasc Imaging. 2017;10:1016-27.

\section{Publisher's Note}

Springer Nature remains neutral with regard to jurisdictional claims in published maps and institutional affiliations.

Ready to submit your research? Choose BMC and benefit from

- fast, convenient online submission

- thorough peer review by experienced researchers in your field

- rapid publication on acceptance

- support for research data, including large and complex data types

- gold Open Access which fosters wider collaboration and increased citations

- maximum visibility for your research: over 100M website views per year

At BMC, research is always in progress.

Learn more biomedcentral.com/submissions 